\title{
Diffusion of Innovation in Two-Sided Markets
}

\author{
Ka Hung Hui, Vijay Subramanian, Dongning Guo and Randall Berry \\ Department of Electrical Engineering and Computer Science \\ Northwestern University, Evanston, IL 60208, USA
}

\begin{abstract}
We consider the diffusion of innovation in twosided markets where both sides choose between an incumbent technology and an innovation. Each player chooses whether to adopt the innovation or not, and repeatedly learns how many players on the other side of the market adopted the innovation and revises her decision accordingly. Using largesystem analysis, we characterize the dynamics of the market, and show a phase transition result: If the initial proportion of adoptors on both sides are sufficiently large, then the innovation will spread to the entire market; otherwise, no one will adopt the innovation. Assuming the innovator can select the proportion of initial adoptors on both sides of the market by advertisements, we study the following three economic problems that are of interest to the innovator: 1) minimizing the advertisement cost while allowing the innovation to spread, 2) minimizing the total cost of advertising and technology improvement while allowing the innovation to spread, and 3) maximizing the total revenue less advertisement cost, where the innovator derives revenue per unit time from each player adopting the innovation. Our analysis provides insight into the types of advertising strategies that can lead to the successful adoption of an innovation.
\end{abstract}

\section{INTRODUCTION}

We consider the diffusion of innovation in two-sided markets. Specifically, we consider a market in which there are two types of players both of whom can choose one of two actions, denoted as the 'status quo' and the 'innovation.' Only players of different types interact with each other, i.e., a type-1 player repeatedly learns the actions of all type- 2 players, and then updates her action to maximize her payoff, and vice versa. This finds applications in the adoption of new technologies. As an example, floppy disks were once used by computer users as external storage, and computer producers included floppy disk drives in desktop computers to read and write data on floppy disks. When new storage media like compact discs (CD) were invented, computer users could switch to this new technology if more computers were equipped with $\mathrm{CD}$ drives, and vice versa. Diffusion of innovation can also be applied to public policy. As another example, electric cars have been introduced to the automobile market, which save gasoline and reduce greenhouse gas emissions. Drivers can choose to buy either electric cars or conventional gasoline cars, and gas stations may invest in electric car charging booths. Drivers and stations prefer coordinating with each other. Subsidies can be used to bring both sides of the market to adopt electric cars. More examples can be found in [1], [2].

Diffusion of innovation through a network has received much attention in, e.g., [3]-[15]. Those references focus on models in which there is a single type of agent, each of whom makes adoption decisions based on her neighbors in an underlying network. This differs from the two-sided setting we are interested in, where there are two types of agents and decisions are driven by the adoptions of the opposite type. For a two-sided market, we use large-system analysis to characterize the dynamics of the market and show the following phase transition result: There is a continuum of threshold pairs such that, if the proportions of initial adopters on both sides are larger than some threshold pair, then the innovation will spread, otherwise the players will eventually give up the innovation. Also, there is a tradeoff between the thresholds for the proportions of initial adopters on both sides: If the threshold on one side increases, then the corresponding threshold on the other side decreases.

Diffusion of innovation is usually formulated as a graphical game [16], where players are assumed to be connected by a network, and their payoffs depend on the decisions of their neighbors, e.g., [4]-[6], [8]-[11], [13]-[15]. Our work is a special case of graphical games, where the underlying network is a complete bipartite graph. This is suitable when the identity of the players is immaterial.

An innovation does not spread simply because of its superiority over the status quo, e.g., the Dvorak keyboard fails to replace the conventional QWERTY keyboard despite having a higher typing efficiency [17]. Therefore, selecting the initial state, or the proportion of initial adopters, is crucial. The initial state can be influenced by advertisements, which encourage players to adopt the innovation. Alternately, the innovator may subsidize players who adopt the innovation, or provide training to help players adopt the innovation. Here, we consider three related economic problems: 1) minimizing the advertisement cost while allowing the innovation to spread, 2) minimizing the total cost of advertising and technology improvement while allowing the innovation to spread, assuming the underlying technology can be improved, and 3) maximizing the total revenue less advertisement cost, where the innovator derives revenue from each player adopting it. These problems are solved assuming linear or logarithmic costs. The solutions to these problems suggest advertising strategies for an innovator, i.e., whether advertisements should target players on only one side or both sides of the market, and how many initial adoptors the innovation should have. Our results indicate that sometimes it is the best to advertise on both sides of the market, which contrasts with the models in [1], [2] where it is optimal to only advertise on one side.

The remainder of the paper is organized as follows. 
Section II reviews related works. The system model is described in Section III. The condition on the initial state for the innovation to spread is discussed in Section IV. The economic problems stated above are discussed in Sections V, VI and VII, respectively. Section VIII concludes the paper.

\section{RELATED WORK}

Many existing works on diffusion of innovation assume that a coordination game with a risk-dominant strategy is played between homogeneous players. The case where players revise their decisions following noisy best-response dynamics was considered in [3]-[7], i.e., players prefer their best response, but do not always follow it. Reference [3] showed that noisy best-response dynamics with global interaction result in every player choosing the risk-dominant strategy. Similar results were obtained in [4], assuming players are located on a lattice, and they interact locally. It was shown in [5] that noisy best-response dynamics converge fast for local interaction but extremely slowly for global interaction. Reference [6] characterized the expected waiting time until all players choose the risk-dominant strategy under noisy best-response dynamics on random graphs. When the degree of risk-dominance exceeds a threshold, the riskdominant strategy spreads under noisy best-response dynamics and gobal interaction, and the expected time for this to happen is bounded independently of the system size [7].

Best-response dynamics were considered in [8]-[15]. In [8], the condition for a strategy to spread from a finite group of population via best-response dynamics with local interaction was characterized. The expected number of adopters of the risk-dominant strategy was characterized in [14]. The results in [4] were extended in [9] to best-response dynamics. It is shown that, the state the system converges to critically depends on the initial state, and its convergence with global interaction is faster than that with local interaction, contrary to [5]. Mean-field approximation was used in [10] to show that for random networks, there exists a threshold for the degree of risk-dominance of a strategy, under which the strategy spreads. A similar approach was used in [11] to characterize the equilibria, and study their stability and their effect on diffusion, where players may incur some random cost when adopting a strategy. In [12], different ways for diffusion to happen were discussed, assuming different players may adopt at a different rate. In [13], compatibility between strategies was considered by allowing players to adopt both strategies with an additional cost. Reference [15] characterized the threshold for the degree of risk-dominance, under which the risk-dominant strategy spreads by letting a single player adopt it initially.

Some recent works consider dynamics on two-sided markets. In [18], it is assumed that when a new player joins one side of the market, she adopts a strategy with a probability depending on the proportion of the players on the other side that has adopted the strategy, and the equilibrium of this dynamics is characterized. It is shown in [19] that the riskdominant strategy is stochastically stable under any noisy best-response dynamics in a two-sided market.
TABLE I

PAYOFF MATRIX.

\begin{tabular}{|c|c|c|c|}
\hline \multicolumn{2}{|c|}{} & \multicolumn{2}{c|}{ Type-2 } \\
\cline { 3 - 4 } & +1 & -1 \\
\hline \multirow{2}{*}{ Type-1 } & +1 & $\left(y_{1}, y_{2}\right)$ & $\left(x_{1}, z_{2}\right)$ \\
\cline { 2 - 4 } & -1 & $\left(z_{1}, x_{2}\right)$ & $\left(w_{1}, w_{2}\right)$ \\
\hline
\end{tabular}

For diffusion of innovation with advertisements, this has been studied in a one-sided setting in [20]; while in [1] and [21], different models of price allocation for a monopoly and two competing products in a two-sided market are studied.

\section{System Model}

We consider a large population of $N$ type- 1 players and $\alpha N$ type-2 players, where $\alpha>0$. Each player chooses one of two strategies: +1 (innovation) or -1 (status quo). The system state is $\left(p_{1}, p_{2}\right)$, the proportions of type- 1 and type- 2 players choosing +1 . Every player plays a game with each player of the opposite type. The payoff of the game is given in Table I. All type-1 and type-2 players have independent Poisson clocks that tick at a rate of $\lambda_{1}$ and $\lambda_{2}$, respectively. When a clock ticks, the corresponding player best-responds to all players of different type by choosing the strategy that maximizes the sum of the payoffs of all the games she participates in. Assuming ties are broken in favor of +1 , then +1 is the best response for a type- 1 player if and only if

$$
p_{2} \geq \frac{w_{1}-x_{1}}{y_{1}-z_{1}+w_{1}-x_{1}},
$$

and the corresponding condition for a type- 2 player is

$$
p_{1} \geq \frac{w_{2}-x_{2}}{y_{2}-z_{2}+w_{2}-x_{2}} .
$$

Notice that conditions (1) and (2) are symmetric in the subscripts. Throughout this paper, when this situation arises, we will use subscripts $a$ and $b$, and it should be understood as two expressions, one with $a=1, b=2$ and the other $a=2$, $b=1$. These conditions only depend on the payoffs through the differences $w_{a}-x_{a}$ and $y_{a}-z_{a}$. Hence, in the following we will assume $x_{a}=z_{a}=0$ without loss of generality, and the above conditions reduce to

$$
p_{a} \geq \frac{w_{b}}{y_{b}+w_{b}} \triangleq q_{a}
$$

Assume $y_{a}>0$ and $w_{a}>0$, so players prefer coordinating with each other. Strategy +1 is assumed to be risk-dominant, meaning that $q_{1}+q_{2}<1$ [22].

\section{Evolution Trajectory}

Given the initial state $\left(p_{1}, p_{2}\right)$ and condition (3), which determines the best response, the following phase transition result and the state at any time $t \geq 0$ are given as follows.

Theorem 1: Consider the large-system limit as $N \rightarrow \infty$ while keeping $\alpha$ constant. If the initial state is $\left(p_{1}, p_{2}\right)$, then the system converges either to $(1,1)$ or $(0,0)$ with probability 1 , and phase transition occurs along

$$
\frac{1}{\lambda_{a}} \ln \frac{p_{a}}{q_{a}}=\frac{1}{\lambda_{b}} \ln \frac{1-p_{b}}{1-q_{b}}, \text { if } p_{a} \geq q_{a} \text { and } p_{b} \leq q_{b} .
$$


TABLE II

EVOLUTION TRAJECTORY AND INITIAL CONDITION.

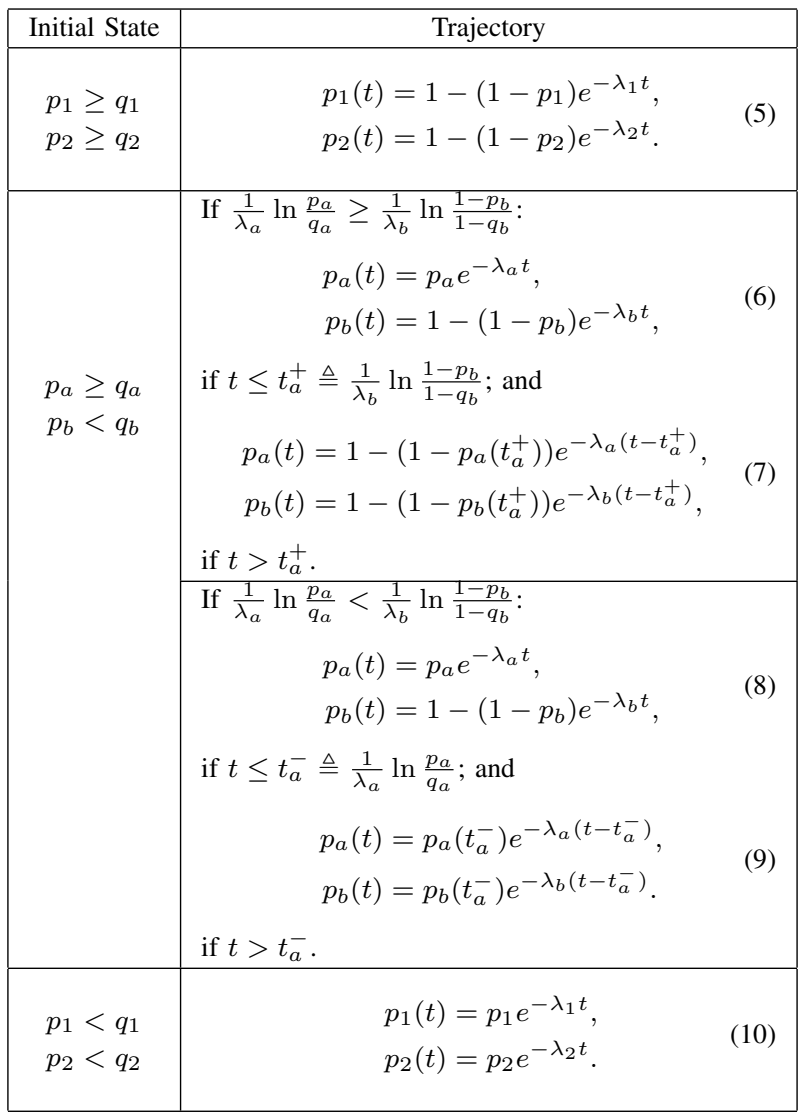

The evolution of the state is given in Table II.

Fig. 1(b) summarizes all possible evolutions, with the initial states marked by crosses. For example, the solid line with a bigger cross is the trajectory (6) and (7) when $a=1$, $b=2$, such that $q_{1}=\frac{1}{3}, q_{2}=\frac{2}{5}, \lambda_{1}=\lambda_{2}=1$, and $\left(p_{1}, p_{2}\right)=\left(\frac{2}{3}, \frac{1}{5}\right)$.

Proof: Depending on whether (3) is satisfied or not, the entire state space is partitioned into four regions as shown in Fig. 1(a).

Let the initial state be $\left(p_{1}, p_{2}\right)=\left(\frac{n_{1}}{N}, \frac{n_{2}}{\alpha N}\right)$, where $n_{1}$ and $n_{2}$ are the initial number of type- 1 and type- 2 players choosing +1 . First assume that $\left(p_{1}, p_{2}\right)$ satisfies (3) for both types of players, i.e., the initial state is in region 1 in Fig. 1(a). Then the best response for any player is +1 , and the state can only change to $\left(p_{1}+\frac{1}{N}, p_{2}\right)$ or $\left(p_{1}, p_{2}+\frac{1}{\alpha N}\right)$, at a (normalized) rate of $\frac{\lambda_{1}\left(N-n_{1}\right)}{N}=\lambda_{1}\left(1-p_{1}\right)$ and $\frac{\lambda_{2}\left(\alpha N-n_{2}\right)}{\alpha N}=\lambda_{2}\left(1-p_{2}\right)$, respectively. By Kurtz's Theorem (Theorem 2.1, Chapter 11 in [23]), if we consider the largesystem limit as $N \rightarrow \infty$ while keeping $\alpha$ constant, the evolution of the state is governed by the differential equations

$$
\frac{d p_{1}(t)}{d t}=\lambda_{1}\left(1-p_{1}(t)\right), \quad \frac{d p_{2}(t)}{d t}=\lambda_{2}\left(1-p_{2}(t)\right) .
$$

Solving these gives (5).

Similarly, if the initial state $\left(p_{1}, p_{2}\right)$ does not satisfy (3) for both types of players, i.e., the initial state is in region 4

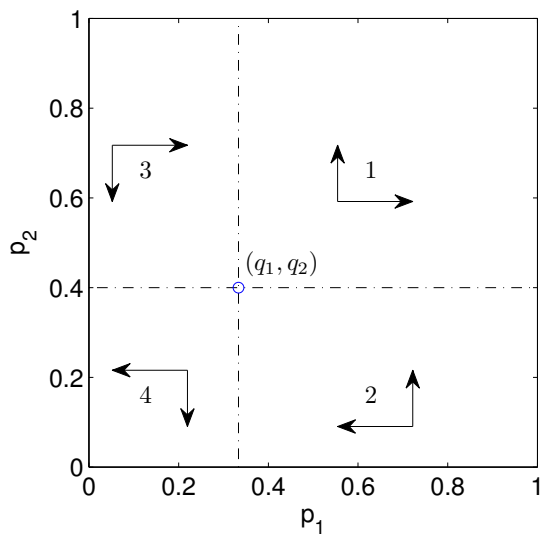

(a)

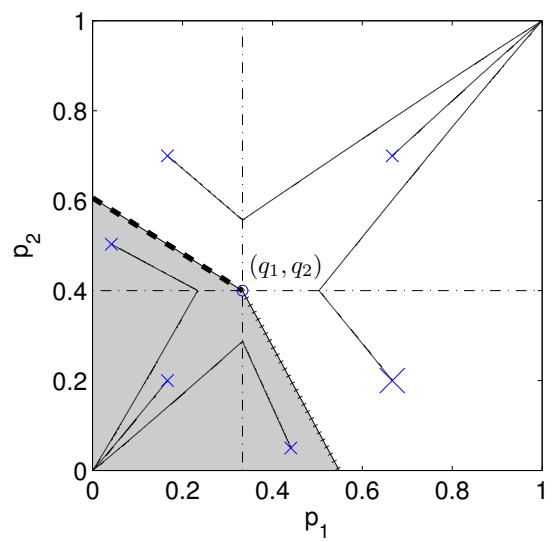

(b)

Fig. 1. The evolution trajectory when $q_{1}=\frac{1}{3}, q_{2}=\frac{2}{5}, \lambda_{1}=\lambda_{2}=1$ : (a) the evolution depends on the initial state, and (b) all possible evolutions in Theorem 1.

in Fig. 1(a), then we have (10).

If the initial state satisfies (3) for only type- $b$ players but not type- $a$ players, i.e., the initial state is in region 2 in Fig. 1(a) if $a=1$, or region 3 in Fig. 1(a) if $a=2$, then the corresponding differential equations are

$$
\frac{d p_{a}(t)}{d t}=\lambda_{a} p_{a}(t), \quad \frac{d p_{b}(t)}{d t}=\lambda_{b}\left(1-p_{b}(t)\right) .
$$

Solving these gives $p_{a}(t)=p_{a} e^{-\lambda_{a} t}$ and $p_{b}(t)=1-$ $\left(1-p_{b}\right) e^{-\lambda_{b} t}$, meaning that eventually either $p_{b}(t)=q_{b}$ and the state enters region 1 , or $p_{a}(t)=q_{a}$ and the state enters region 4 . The time $t_{a}^{+}$such that $p_{b}\left(t_{a}^{+}\right)=q_{b}$ is $t_{a}^{+}=\frac{1}{\lambda_{b}} \ln \frac{1-p_{b}}{1-q_{b}}$, while the time $t_{a}^{-}$such that $p_{a}\left(t_{a}^{-}\right)=q_{a}$ is $t_{a}^{-}=\frac{1}{\lambda_{a}} \ln \frac{p_{a}}{q_{a}}$. If $\frac{1}{\lambda_{a}} \ln \frac{p_{a}}{q_{a}} \geq \frac{1}{\lambda_{b}} \ln \frac{1-p_{b}}{1-q_{b}}$, then $t_{a}^{-} \geq t_{a}^{+}$, and the state enters region 1, therefore we have (6) and (7). Otherwise, $t_{a}^{-}<t_{a}^{+}$, and the state enters region 4 , hence we have (8) and (9).

The result of Theorem 1 is illustrated in Fig. 2. The phase transition boundary (4) is represented by dotted lines when $a=1, b=2$, and by dashed lines when $a=2, b=1$. The unshaded region, including the phase transition boundary, is the basin of attraction of $(1,1)$, i.e., if the initial state 


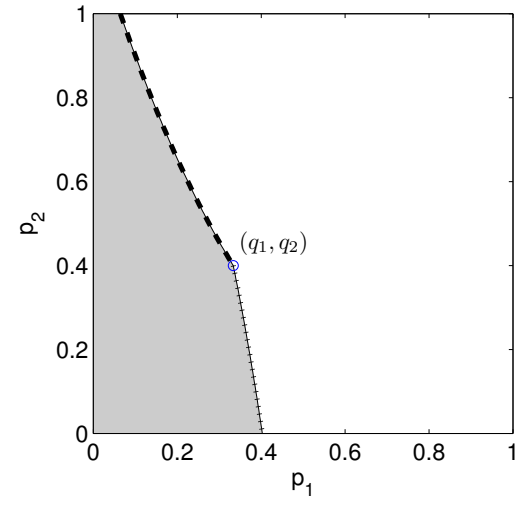

(a)

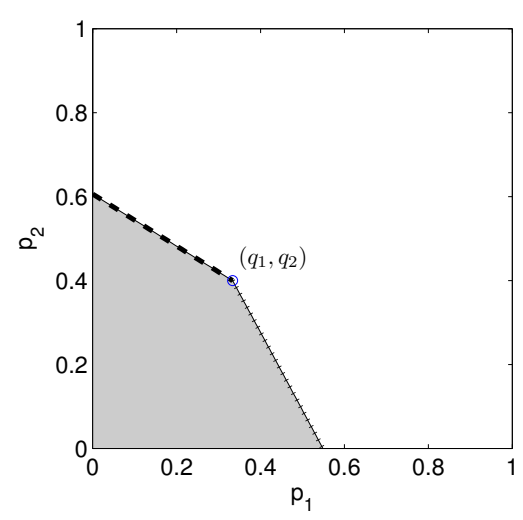

(b)

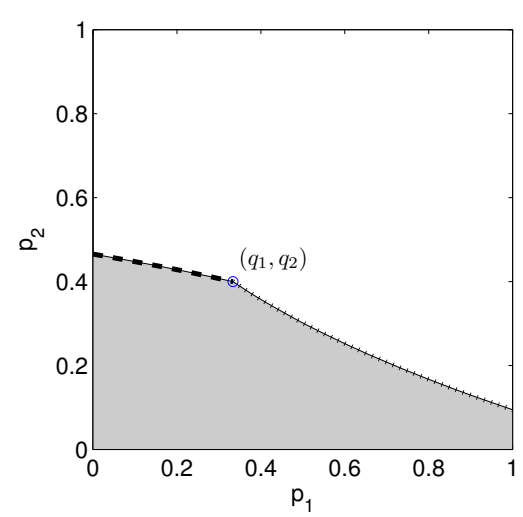

(c)

Fig. 2. Basins of attraction when $q_{1}=\frac{1}{3}, q_{2}=\frac{2}{5}$ and (a) $\frac{\lambda_{1}}{\lambda_{2}}<\frac{\ln \left(1-q_{1}\right)}{\ln q_{2}}$, (b) $\frac{\ln \left(1-q_{1}\right)}{\ln q_{2}} \leq \frac{\lambda_{1}}{\lambda_{2}} \leq \frac{\ln q_{1}}{\ln \left(1-q_{2}\right)}$, and (c) $\frac{\lambda_{1}}{\lambda_{2}}>\frac{\ln q_{1}}{\ln \left(1-q_{2}\right)}$.

is inside this region, the system converges to $(1,1)$ with probability 1 . The shaded region is the basin of attraction of $(0,0)$. If $(0,1)$ lies in the basin of attraction of $(0,0)$, this implies that even if all type- 2 players initially choose the risk-dominant strategy +1 , all of them will switch to -1 eventually. This is possible if type- 2 players update more frequently than type-1 players, i.e., $\lambda_{1}$ and $\lambda_{2}$ have to satisfy

$$
\frac{\lambda_{1}}{\lambda_{2}}<\frac{\ln \left(1-q_{1}\right)}{\ln q_{2}} .
$$

This is illustrated in Fig. 2(a). Similarly, if $(1,0)$ lies in the basin of attraction of $(0,0)$, then type- 1 players update more frequently and it is possible that even though initially all type- 1 players choose the risk-dominant strategy +1 , eventually they all switch to -1 . This happens when

$$
\frac{\lambda_{1}}{\lambda_{2}}>\frac{\ln q_{1}}{\ln \left(1-q_{2}\right)} .
$$

This is illustrated in Fig. 2(c). The remaining case where

$$
\frac{\ln \left(1-q_{1}\right)}{\ln q_{2}} \leq \frac{\lambda_{1}}{\lambda_{2}} \leq \frac{\ln q_{1}}{\ln \left(1-q_{2}\right)}
$$

is shown in Fig. 2(b). Here type-1 and type-2 players update at similar rates. If all players of the same type initially choose the risk-dominant strategy +1 , then eventually all players will choose +1 .

\section{Cost Minimization with Advertisement}

It has been established in Section IV that for an innovation to spread, the initial proportion of adopters must be sufficiently large, i.e., the initial state has to be inside the basin of attraction of $(1,1)$. Suppose the innovator advertises to encourage players to adopt the innovation, such that by spending a cost $C\left(p_{1}, p_{2}\right)$, a proportion $p_{1}$ of type-1 players and a proportion $p_{2}$ of type- 2 players adopt the innovation. Then the question is how the innovator should choose $p_{1}$ and $p_{2}$ so that the system will reach $(1,1)$ while the advertisement cost is minimized.
Assume that by spending $C_{a}$ on advertisements targeting at type- $a$ players, a proportion $p_{a}=F_{a}\left(C_{a}\right)$ of type$a$ players switch to +1 , where $F_{a}(\cdot)$ is increasing. The advertisement cost is $C\left(p_{1}, p_{2}\right)=C_{1}+C_{2}=F_{1}^{-1}\left(p_{1}\right)+$ $F_{2}^{-1}\left(p_{2}\right)$. This additive cost model is appropriate if different types of players focus on different aspects of the innovation. Since $F_{a}(\cdot)$ is increasing, $F_{a}^{-1}(\cdot)$ is also increasing, and the minimizing state must lie on (4). The minimal advertisement cost is then given by the following optimization:

$$
\begin{array}{ll}
\text { minimize } & F_{a}^{-1}\left(p_{a}\right)+F_{b}^{-1}\left(p_{b}\right) \\
\text { subject to } & \frac{1}{\lambda_{a}} \ln \frac{p_{a}}{q_{a}}=\frac{1}{\lambda_{b}} \ln \frac{1-p_{b}}{1-q_{b}} \\
& p_{a} \geq q_{a} \\
& p_{b} \leq q_{b} .
\end{array}
$$

This can be solved by first transforming the problem into

$$
\begin{array}{ll}
\text { minimize } & F_{a}^{-1}\left(q_{a} e^{-\lambda_{a} s}\right)+F_{b}^{-1}\left(1-\left(1-q_{b}\right) e^{-\lambda_{b} s}\right) \\
\text { subject to } & \frac{1}{\lambda_{b}} \ln \left(1-q_{b}\right) \leq s \leq 0
\end{array}
$$

if $\frac{\lambda_{a}}{\lambda_{b}} \leq \frac{\ln q_{a}}{\ln \left(1-q_{b}\right)}$, or

$$
\begin{array}{ll}
\text { minimize } & F_{a}^{-1}\left(q_{a} e^{-\lambda_{a} s}\right)+F_{b}^{-1}\left(1-\left(1-q_{b}\right) e^{-\lambda_{b} s}\right) \\
\text { subject to } & \frac{1}{\lambda_{a}} \ln q_{a} \leq s \leq 0
\end{array}
$$

if $\frac{\lambda_{a}}{\lambda_{b}}>\frac{\ln q_{a}}{\ln \left(1-q_{b}\right)}$, using the parametrization

$$
p_{a}=q_{a} e^{-\lambda_{a} s}, \quad p_{b}=1-\left(1-q_{b}\right) e^{-\lambda_{b} s}
$$

and then followed by differentiation. Examples for linear and logarithmic costs are given below.

\section{A. Linear Cost}

Assume a linear advertisement cost $C_{a}=F_{a}^{-1}\left(p_{a}\right)=$ $\gamma_{a} p_{a}$, where $\gamma_{a}>0$. The solution to minimization problem (11) depends on $\lambda_{1}, \lambda_{2}, \gamma_{1}$ and $\gamma_{2}$ through the ratios $\frac{\lambda_{1}}{\lambda_{2}}$ and $\frac{\gamma_{1}}{\gamma_{2}}$ only. Without loss of generality, let $\lambda_{1}=\lambda, \lambda_{2}=1$, $\gamma_{1}=\gamma$ and $\gamma_{2}=1$. Let $C_{\min }^{1}\left(q_{1}, q_{2}, \lambda, \gamma\right)$ be the minimum cost given by (11) when $a=1, b=2$ and $C_{\min }^{2}\left(q_{1}, q_{2}, \lambda, \gamma\right)$ 
TABLE III

LineAR AdVERTISEMENT Cost.

\begin{tabular}{|c|c|c|}
\hline & $C_{\min }^{1}$ and the corresponding $\left(p_{1}^{*}, p_{2}^{*}\right)$ & $C_{\min }^{2}$ and the corresponding $\left(p_{1}^{*}, p_{2}^{*}\right)$ \\
\hline$\lambda>\frac{\ln q_{1}}{\ln \left(1-q_{2}\right)}$ & $\begin{array}{l}\text { If } \gamma \geq \frac{1-q_{2}}{\lambda q_{1}}: C_{\min }^{1}=\gamma q_{1}+q_{2}, \text { at }\left(q_{1}, q_{2}\right) \\
\text { if } \frac{1-q_{2}}{\lambda q_{1}} \geq \gamma \geq \frac{1-q_{2}}{\lambda q_{1}^{\frac{1}{\lambda}}}: \\
C_{\min }^{1}=1-\left(1-\frac{1}{\lambda}\right)\left[\frac{\left(1-q_{2}\right)^{\lambda}}{\gamma \lambda q_{1}}\right]^{\frac{1}{\lambda-1}}, \text { at }\left(p_{1}^{*}, p_{2}^{*}\right) \text { such } \\
\text { that } \frac{1}{\lambda} \ln \frac{p_{1}^{*}}{q_{1}}=\ln \frac{1-p_{2}^{*}}{1-q_{2}} \text { and } \gamma \lambda=\frac{1-p_{2}^{*}}{p_{1}^{*}} ; \\
\text { if } \gamma \leq \frac{1-q_{2}}{\lambda q_{1}^{\frac{1}{\lambda}}}: C_{\min }^{1}=\gamma+1-\frac{1-q_{2}}{q_{1}^{\frac{1}{\lambda}}}, \text { at }\left(1,1-\frac{1-q_{2}}{q_{1}^{\frac{1}{\lambda}}}\right)\end{array}$ & \multirow{2}{*}{$\begin{array}{l}\text { If } \gamma \leq \frac{q_{2}}{q_{1}} \frac{1-\left(1-q_{1}\right)^{\frac{1}{\lambda}}}{\left(1-q_{1}\right)^{\frac{1}{\lambda}}}: C_{\min }^{2}=\gamma q_{1}+q_{2}, \text { at }\left(q_{1}, q_{2}\right) \\
\text { if } \gamma \geq \frac{q_{2}}{q_{1}} \frac{1-\left(1-q_{1}\right)^{\frac{1}{\lambda}}}{\left(1-q_{1}\right)^{\frac{1}{\lambda}}}: \\
C_{\min }^{2}=\frac{q_{2}}{\left(1-q_{1}\right)^{\frac{1}{\lambda}}}, \text { at }\left(0, \frac{q_{2}}{\left(1-q_{1}\right)^{\frac{1}{\lambda}}}\right)\end{array}$} \\
\hline $1<\lambda \leq \frac{\ln q_{1}}{\ln \left(1-q_{2}\right)}$ & $\begin{array}{l}\text { If } \gamma \geq \frac{1-q_{2}}{\lambda q_{1}}: C_{\min }^{1}=\gamma q_{1}+q_{2}, \text { at }\left(q_{1}, q_{2}\right) \\
\text { if } \frac{1-q_{2}}{\lambda q_{1}} \geq \gamma \geq \frac{\left(1-q_{2}\right)^{\lambda}}{\lambda q_{1}}: \\
C_{\min }^{1}=1-\left(1-\frac{1}{\lambda}\right)\left[\frac{\left(1-q_{2}\right)^{\lambda}}{\gamma \lambda q_{1}}\right]^{\frac{1}{\lambda-1}} \text {, at }\left(p_{1}^{*}, p_{2}^{*}\right) \text { such } \\
\text { that } \frac{1}{\lambda} \ln \frac{p_{1}^{*}}{q_{1}}=\ln \frac{1-p_{2}^{*}}{1-q_{2}} \text { and } \gamma \lambda=\frac{1-p_{2}^{*}}{p_{1}^{*}} \\
\text { if } \gamma \leq \frac{\left(1-q_{2}\right)^{\lambda}}{\lambda q_{1}}: C_{\min }^{1}=\frac{\gamma q_{1}}{\left(1-q_{2}\right)^{\lambda}} \text {, at }\left(\frac{q_{1}}{\left(1-q_{2}\right)^{\lambda}}, 0\right) \text {. }\end{array}$ & \\
\hline$\lambda=1$ & $\begin{array}{l}\text { If } \gamma \geq \frac{1-q_{2}}{q_{1}}: C_{\min }^{1}=\gamma q_{1}+q_{2}, \text { at }\left(q_{1}, q_{2}\right) \\
\text { if } \gamma \leq \frac{1-q_{2}}{q_{1}}: C_{\min }^{1}=\frac{\gamma q_{1}}{1-q_{2}}, \text { at }\left(\frac{q_{1}}{1-q_{2}}, 0\right)\end{array}$ & $\begin{array}{l}\text { If } \gamma \leq \frac{q_{2}}{1-q_{1}}: C_{\min }^{2}=\gamma q_{1}+q_{2}, \text { at }\left(q_{1}, q_{2}\right) \\
\text { if } \gamma \geq \frac{q_{2}}{1-q_{1}}: C_{\min }^{2}=\frac{q_{2}}{1-q_{1}}, \text { at }\left(0, \frac{q_{2}}{1-q_{1}}\right)\end{array}$ \\
\hline $1>\lambda \geq \frac{\ln \left(1-q_{1}\right)}{\ln q_{2}}$ & \multirow[t]{2}{*}{$\begin{array}{l}\text { If } \gamma \geq \frac{q_{2}}{q_{1}} \frac{\left(1-q_{2}\right)^{\lambda}}{1-\left(1-q_{2}\right)^{\lambda}}: C_{\min }^{1}=\gamma q_{1}+q_{2} \text {, at }\left(q_{1}, q_{2}\right) \\
\text { if } \gamma \leq \frac{q_{2}}{q_{1}} \frac{\left(1-q_{2}\right)^{\lambda}}{1-\left(1-q_{2}\right)^{\lambda}}: \\
C_{\min }^{1}=\frac{\gamma q_{1}}{\left(1-q_{2}\right)^{\lambda}}, \text { at }\left(\frac{q_{1}}{\left(1-q_{2}\right)^{\lambda}}, 0\right)\end{array}$} & $\begin{array}{l}\text { If } \gamma \leq \frac{q_{2}}{\lambda\left(1-q_{1}\right)}: C_{\min }^{2}=\gamma q_{1}+q_{2}, \text { at }\left(q_{1}, q_{2}\right) \\
\text { if } \frac{q_{2}}{\lambda\left(1-q_{1}\right)} \leq \gamma \leq \frac{q_{2}}{\lambda\left(1-q_{1}\right)^{\frac{1}{\lambda}}}: \\
C_{\min }^{2}=\gamma-(1-\lambda)\left[\frac{\gamma \lambda^{\lambda}\left(1-q_{1}\right)}{q_{2}^{\lambda}}\right]^{\frac{1}{1-\lambda}} \text {, at }\left(p_{1}^{*}, p_{2}^{*}\right) \text { such } \\
\text { that } \frac{1}{\lambda} \ln \frac{1-p_{1}^{*}}{1-q_{1}}=\ln \frac{p_{2}^{*}}{q_{2}} \text { and } \gamma \lambda=\frac{p_{2}^{*}}{1-p_{1}^{*}} ; \\
\text { if } \gamma \geq \frac{q_{2}}{\lambda\left(1-q_{1}\right)^{\frac{1}{\lambda}}}: C_{\min }^{2}=\frac{q_{2}}{\left(1-q_{1}\right)^{\frac{1}{\lambda}}} \text {, at }\left(0, \frac{q_{2}}{\left(1-q_{1}\right)^{\frac{1}{\lambda}}}\right) \text {. }\end{array}$ \\
\hline$\lambda<\frac{\ln \left(1-q_{1}\right)}{\ln q_{2}}$ & & $\begin{array}{l}\text { If } \gamma \leq \frac{q_{2}}{\lambda\left(1-q_{1}\right)}: C_{\min }^{2}=\gamma q_{1}+q_{2}, \text { at }\left(q_{1}, q_{2}\right) \text {; } \\
\text { if } \frac{q_{2}}{\lambda\left(1-q_{1}\right)} \leq \gamma \leq \frac{q_{2}^{\lambda}}{\lambda\left(1-q_{1}\right)}: \\
C_{\min }^{2}=\gamma-(1-\lambda)\left[\frac{\gamma \lambda^{\lambda}\left(1-q_{1}\right)}{q_{2} \lambda}\right]^{\frac{1}{1-\lambda}} \text {, at }\left(p_{1}^{*}, p_{2}^{*}\right) \text { such } \\
\text { that } \frac{1}{\lambda} \ln \frac{1-p_{1}^{*}}{1-q_{1}}=\ln \frac{p_{2}^{*}}{q_{2}} \text { and } \gamma \lambda=\frac{p_{2}^{*}}{1-p_{1}^{*}} ; \\
\text { if } \gamma \geq \frac{q_{2}^{\lambda}}{\lambda\left(1-q_{1}\right)}: \\
C_{\min }^{2}=\gamma\left(1-\frac{1-q_{1}}{q_{2}^{\lambda}}\right)+1, \text { at }\left(1-\frac{1-q_{1}}{q_{2}^{\lambda}}, 1\right) \text {. }\end{array}$ \\
\hline
\end{tabular}

be the minimum cost given by (11) when $a=2, b=1$. Then the minimum cost is $C_{\min }=\min \left(C_{\min }^{1}, C_{\min }^{2}\right)$.

By solving (12)-(13), we obtain the following lemma.

Lemma 1: $C_{\mathrm{min}}^{1}, C_{\mathrm{min}}^{2}$ and the corresponding minimizing state $\left(p_{1}^{*}, p_{2}^{*}\right)$ are given in Table III.

In general, as the cost ratio $\gamma$ increases, the minimizing state $\left(p_{1}^{*}, p_{2}^{*}\right)$ changes such that $p_{1}^{*}$ decreases and $p_{2}^{*}$ increases. Based on Lemma 1, we arrive at the following strategy for linear advertisement cost.

Theorem 2: The following advertising strategy minimizes the linear advertisement cost:

1) Only target type-1 players if

a) $1 \leq \lambda \leq \frac{\ln q_{1}}{\ln \left(1-q_{2}\right)}, \gamma \leq \frac{\left(1-q_{2}\right)^{\lambda}}{\lambda q_{1}}$ and $C_{\min }^{1} \leq C_{\min }^{2}$, or

b) $\lambda<1, \gamma \leq \frac{q_{2}}{q_{1}} \frac{\left(1-q_{2}\right)^{\lambda}}{1-\left(1-q_{2}\right)^{\lambda}}$ and $C_{\min }^{1} \leq C_{\min }^{2}$;

2) Only target type- 2 players if

a) $\lambda>1, \gamma \geq \frac{q_{2}}{q_{1}} \frac{1-\left(1-q_{1}\right)^{\frac{1}{\lambda}}}{\left(1-q_{1}\right)^{\frac{1}{\lambda}}}$ and $C_{\min }^{2} \leq C_{\min }^{1}$, or

b) $1 \geq \lambda \geq \frac{\ln \left(1-q_{1}\right)}{\ln q_{2}}, \gamma \geq \frac{q_{2}}{\lambda\left(1-q_{1}\right)^{\frac{1}{\lambda}}}$ and $C_{\min }^{2} \leq C_{\min }^{1}$;
3) Target both type-1 and type- 2 players if

a) $\lambda>\frac{\ln q_{1}}{\ln \left(1-q_{2}\right)}, \frac{1-q_{2}}{\lambda q_{1}} \geq \gamma \geq \frac{1-q_{2}}{\lambda q_{1}^{\frac{1}{\lambda}}}$ and $C_{\min }^{1} \leq C_{\min }^{2}$,

b) $1<\lambda \leq \frac{\ln q_{1}}{\ln \left(1-q_{2}\right)}, \frac{1-q_{2}}{\lambda q_{1}} \geq \gamma \geq \frac{\left(1-q_{2}\right)^{\lambda}}{\lambda q_{1}}$ and $C_{\min }^{1} \leq$ $C_{\text {min }}^{2}$, or

c) $1>\lambda \geq \frac{\ln \left(1-q_{1}\right)}{\ln q_{2}}, \frac{q_{2}}{\lambda\left(1-q_{1}\right)} \leq \gamma \leq \frac{q_{2}}{\lambda\left(1-q_{1}\right)^{\frac{1}{\lambda}}}$ and $C_{\min }^{2} \leq C_{\min }^{1}$, or

d) $\lambda<\frac{\ln \left(1-q_{1}\right)}{\ln q_{2}}, \frac{q_{2}}{\lambda\left(1-q_{1}\right)} \leq \gamma \leq \frac{q_{2}^{\lambda}}{\lambda\left(1-q_{1}\right)}$ and $C_{\min }^{2} \leq$ $C_{\mathrm{min}}^{1}$

4) Target all type-1 players and some type-2 players if $\lambda>\frac{\ln q_{1}}{\ln \left(1-q_{2}\right)}, \gamma \leq \frac{1-q_{2}}{\lambda q_{1}^{\frac{1}{\lambda}}}$ and $C_{\min }^{1} \leq C_{\min }^{2} ;$

5) Target all type- 2 players and some type- 1 players if $\lambda<\frac{\ln \left(1-q_{1}\right)}{\ln q_{2}}, \gamma \geq \frac{q_{2}^{\lambda}}{\lambda\left(1-q_{1}\right)}$ and $C_{\min }^{2} \leq C_{\min }^{1}$.

In a typical two-sided market, one side is treated as a profit center and the other side a loss leader, therefore advertisements only target the loss leader [1], [2]. While sufficient advertisements targeting only one type of players allow the innovation to take over the market (see Fig. 2), 
TABLE IV

Logarithmic Advertisement Cost.

\begin{tabular}{|c|c|c|}
\hline & $C_{\min }^{1}$ and the corresponding $\left(p_{1}^{*}, p_{2}^{*}\right)$ & $C_{\min }^{2}$ and the corresponding $\left(p_{1}^{*}, p_{2}^{*}\right)$ \\
\hline$\lambda>\frac{\ln q_{1}}{\ln \left(1-q_{2}\right)}$ & $\begin{array}{l}\text { If } \frac{\mu}{\lambda} \leq \frac{q_{1}}{1-q_{1}}: \\
C_{\min }^{1}=-\frac{1}{\mu} \ln \left(1-q_{1}\right)-\ln \left(1-q_{2}\right), \text { at }\left(q_{1}, q_{2}\right) ; \\
\text { if } \frac{\mu}{\lambda} \geq \frac{q_{1}}{1-q_{1}}: \\
C_{\min }^{1}=-\ln \frac{1-q_{2}}{q_{1}^{\frac{1}{\lambda}}}+\left(\frac{1}{\lambda}+\frac{1}{\mu}\right) h_{2}\left(\frac{\mu}{\lambda+\mu}\right) \\
\text { at }\left(\frac{\mu}{\lambda+\mu}, 1-\frac{1-q_{2}}{q_{1}^{\frac{1}{\lambda}}}\left(\frac{\mu}{\lambda+\mu}\right)^{\frac{1}{\lambda}}\right)\end{array}$ & $\begin{array}{l}\text { If } \frac{\mu}{\lambda} \geq \frac{1-q_{2}}{q_{2}}: \\
C_{\min }^{2}=-\frac{1}{\mu} \ln \left(1-q_{1}\right)-\ln \left(1-q_{2}\right), \text { at }\left(q_{1}, q_{2}\right) \text {; } \\
\text { if } \frac{\left(1-q_{1}\right)^{\frac{1}{\lambda}}-q_{2}}{q_{2}} \leq \frac{\mu}{\lambda} \leq \frac{1-q_{2}}{q_{2}}: \\
C_{\min }^{2}=-\frac{1}{\mu} \ln \frac{1-q_{1}}{q_{2}^{\lambda}}+\left(1+\frac{\lambda}{\mu}\right) h_{2}\left(\frac{\mu}{\lambda+\mu}\right), \\
\text { at }\left(1-\frac{1-q_{1}}{q_{2}^{\lambda}}\left(\frac{\lambda}{\lambda+\mu}\right)^{\lambda}, \frac{\lambda}{\lambda+\mu}\right) ;\end{array}$ \\
\hline$\frac{\ln \left(1-q_{1}\right)}{\ln q_{2}} \leq \lambda \leq \frac{\ln q_{1}}{\ln \left(1-q_{2}\right)}$ & $\begin{array}{l}\text { If } \frac{\mu}{\lambda} \leq \frac{q_{1}}{1-q_{1}}: \\
C_{\min }^{1}=-\frac{1}{\mu} \ln \left(1-q_{1}\right)-\ln \left(1-q_{2}\right), \text { at }\left(q_{1}, q_{2}\right) \\
\text { if } \frac{q_{1}}{1-q_{1}} \leq \frac{\mu}{\lambda} \leq \frac{q_{1}}{\left(1-q_{2}\right)^{\lambda}-q_{1}}\end{array}$ & if $\begin{aligned} \frac{\mu}{\lambda} & \leq \frac{\left(1-q_{1}\right)^{\frac{1}{\lambda}}-q_{2}}{q_{2}}: \\
C_{\min }^{2} & =-\ln \left[1-\frac{q_{2}}{\left(1-q_{1}\right)^{\frac{1}{\lambda}}}\right] \text {, at }\left(0, \frac{q_{2}}{\left(1-q_{1}\right)^{\frac{1}{\lambda}}}\right) .\end{aligned}$ \\
\hline$\lambda<\frac{\ln \left(1-q_{1}\right)}{\ln q_{2}}$ & $\begin{array}{l}C_{\min }^{1}=-\ln \frac{1-q_{2}}{q_{1}^{\frac{1}{\lambda}}}+\left(\frac{1}{\lambda}+\frac{1}{\mu}\right) h_{2}\left(\frac{\mu}{\lambda+\mu}\right), \\
\text { at }\left(\frac{\mu}{\lambda+\mu}, 1-\frac{1-q_{2}}{q_{1}^{\frac{1}{\lambda}}}\left(\frac{\mu}{\lambda+\mu}\right)^{\frac{1}{\lambda}}\right) ; \\
\text { if } \frac{\mu}{\lambda} \geq \frac{q_{1}}{\left(1-q_{2}\right)^{\lambda}-q_{1}}: \\
C_{\min }^{1}=-\frac{1}{\mu} \ln \left[1-\frac{q_{1}}{\left(1-q_{2}\right)^{\lambda}}\right] \text {, at }\left(\frac{q_{1}}{\left(1-q_{2}\right)^{\lambda}}, 0\right) .\end{array}$ & $\begin{array}{l}\text { If } \frac{\mu}{\lambda} \geq \frac{1-q_{2}}{q_{2}}: \\
C_{\min }^{2}=-\frac{1}{\mu} \ln \left(1-q_{1}\right)-\ln \left(1-q_{2}\right), \text { at }\left(q_{1}, q_{2}\right) ; \\
\text { if } \frac{\mu}{\lambda} \leq \frac{1-q_{2}}{q_{2}}: \\
C_{\min }^{2}=-\frac{1}{\mu} \ln \frac{1-q_{1}}{q_{2}^{\lambda}}+\left(1+\frac{\lambda}{\mu}\right) h_{2}\left(\frac{\mu}{\lambda+\mu}\right), \\
\text { at }\left(1-\frac{1-q_{1}}{q_{2}^{\lambda}}\left(\frac{\lambda}{\lambda+\mu}\right)^{\lambda}, \frac{\lambda}{\lambda+\mu}\right) .\end{array}$ \\
\hline
\end{tabular}

Theorem 2 states that such approach is not always costefficient: Sometimes it is the best to advertise on both sides, except when $\lambda=1$.

The evolution resulting from minimizing the advertisement cost is never monotone, i.e., either $p_{1}(t)$ or $p_{2}(t)$ has to decrease first and then increase to 1 . If it is required that the evolution is monotone, meaning that we minimize $\gamma_{1} p_{1}+\gamma_{2} p_{2}$ subject to $p_{1} \geq q_{1}$ and $p_{2} \geq q_{2}$, obviously the minimum is attained at $\left(q_{1}, q_{2}\right)$. But $\left(q_{1}, q_{2}\right)$ never minimizes the advertisement cost if monotonicity is not required and the advertisement cost is linear. On the other hand, if the $\left(p_{1}, p_{2}\right)$ that minimizes the advertisement cost is chosen to be the initial state, the system will first evolve to $\left(q_{1}, q_{2}\right)$ (according to Theorem 1) and then to $(1,1)$. This shows the tradeoff between advertisement cost and time: advertisement cost is reduced at the expense of spending more time to spread the innovation. As an example, suppose both types of players update once every year, the advertisement cost is $\$ 100$ on either side, $q_{1}=\frac{1}{3}$ and $q_{2}=\frac{2}{5}$. The minimum cost is $\frac{500}{9}$, and the corresponding initial state is $\left(\frac{5}{9}, 0\right)$. If the initial state is chosen to be $\left(q_{1}, q_{2}\right)$, then the cost is increased to $\frac{220}{3}$ and the time saved is approximately half a year.

\section{B. Logarithmic Cost}

Assume a logarithmic advertisement cost $C_{a}=$ $F_{a}^{-1}\left(p_{a}\right)=-\frac{1}{\mu_{a}} \ln \left(1-p_{a}\right)$, where $\mu_{a}>0$. This means $p_{a}=$ $1-e^{-\mu_{a} C_{a}}$, so $p_{a}$ is concave in $C_{a}$, and advertisements have diminishing returns. This cost model means that doubling the advertisement cost makes the same proportion of the remaining population switch to the innovation. So it is impossible to make all players of the same type switch to the innovation by advertisements only. In this case too, the solution to minimization problem (11) depends on $\lambda_{1}, \lambda_{2}$, $\mu_{1}$ and $\mu_{2}$ through the ratios $\frac{\lambda_{1}}{\lambda_{2}}$ and $\frac{\mu_{1}}{\mu_{2}}$ only. Without loss of generality, let $\lambda_{1}=\lambda, \lambda_{2}=1, \mu_{1}=\mu$ and $\mu_{2}=1$. Let $C_{\text {min }}^{1}\left(q_{1}, q_{2}, \lambda, \mu\right)$ be the minimum cost given by (11) when $a=1, b=2$ and $C_{\min }^{2}\left(q_{1}, q_{2}, \lambda, \mu\right)$ be the minimum cost given by (11) when $a=2, b=1$. Then the minimum cost is $C_{\min }=\min \left(C_{\min }^{1}, C_{\min }^{2}\right)$.

By solving (12)-(13), we obtain the following lemma.

Lemma 2: $C_{\min }^{1}, C_{\min }^{2}$ and the corresponding minimizing state $\left(p_{1}^{*}, p_{2}^{*}\right)$ are given in Table IV, where $h_{2}(\cdot)$ in the table is the binary entropy function: $h_{2}(x)=-x \ln x-(1-x) \ln (1-$ $x)$.

Based on Lemma 2, we arrive at the following strategy for logarithmic advertisement cost.

Theorem 3: The following advertising strategy minimizes the logarithmic advertisement cost:

1) Only target type-1 players if $\lambda \leq \frac{\ln q_{1}}{\ln \left(1-q_{2}\right)}, \frac{\mu}{\lambda} \geq$ $\frac{q_{1}}{\left(1-q_{2}\right)^{\lambda}-q_{1}}$ and $C_{\min }^{1} \leq C_{\min }^{2}$

2) Only target type-2 players if $\lambda \geq \frac{\ln \left(1-q_{1}\right)}{\ln q_{2}}, \frac{\mu}{\lambda} \leq$ $\frac{\left(1-q_{1}\right)^{\frac{1}{\lambda}}-q_{2}}{q_{2}}$ and $C_{\min }^{2} \leq C_{\min }^{1}$

3) Target both type-1 and type- 2 players in any of the remaining cases.

As with linear cost, Theorem 3 also states that advertisements targeting only one type of players are not always costefficient.

\section{AdVERTISEMENT AND TECHNOLOGy IMPROVEMENT}

In addition to choosing the initial state, suppose the innovator also wants to determine $y_{1}$ and $y_{2}$, i.e., how much it should improve over the status quo. Assume that the innovator spends $C_{a}^{\prime}$ to improve for type- $a$ players so that $y_{a}=G_{a}\left(C_{a}^{\prime}\right)$ where $G_{a}(\cdot)$ is increasing. Then the total cost of technology improvement and advertising is $C=C_{1}+$ $C_{2}+C_{1}^{\prime}+C_{2}^{\prime}=F_{1}^{-1}\left(p_{1}\right)+F_{2}^{-1}\left(p_{2}\right)+G_{1}^{-1}\left(y_{1}\right)+G_{2}^{-1}\left(y_{2}\right)$. To minimize the total cost, the innovator has to solve the 
following minimization problem:

$$
\begin{array}{ll}
\text { minimize } & F_{a}^{-1}\left(p_{a}\right)+F_{b}^{-1}\left(p_{b}\right)+G_{a}^{-1}\left(y_{a}\right)+G_{b}^{-1}\left(y_{b}\right) \\
\text { subject to } & \frac{1}{\lambda_{a}} \ln \frac{p_{a}}{q_{a}}=\frac{1}{\lambda_{b}} \ln \frac{1-p_{b}}{1-q_{b}} \\
& p_{a} \geq q_{a} \\
& p_{b} \leq q_{b} \\
& q_{a}=\frac{w_{b}}{y_{b}+w_{b}} \\
& q_{b}=\frac{w_{a}}{y_{a}+w_{a}} \\
& y_{a} \geq w_{a} \\
y_{b} & \geq w_{b} .
\end{array}
$$

Therefore, minimizing the total cost is nontrivial since a reduction in the cost of technology improvement results in an increase in the values of $q_{1}$ and $q_{2}$, which leads to an increase in the advertisement cost.

In the following assume $y_{a}=G_{a}\left(C_{a}^{\prime}\right)=w_{a}+\beta_{a} C_{a}^{\prime}$, where $\beta_{a}>0$, and a linear advertisement cost as in Section V-A. Then the total cost is $C=\gamma_{1} p_{1}+\gamma_{2} p_{2}+$ $\frac{y_{1}-w_{1}}{\beta_{1}}+\frac{y_{2}-w_{2}}{\beta_{2}}$. For illustrative purposes, assume $\lambda_{1}=$ $\lambda_{2}=1$ and $\gamma_{1}=\gamma_{2}=1$. The following result provides sufficient conditions for the innovator to spend on technology improvement. Recall (3) that $q_{a}=\frac{w_{b}}{y_{b}+w_{b}}$.

Theorem 4: Assume $\lambda_{1}=\lambda_{2}=1$ and $\gamma_{1}=\gamma_{2}=1$. If $2\left(\frac{w_{a}}{\beta_{a}}\right)^{2} \leq \frac{w_{b}}{\beta_{b}} \leq \frac{1}{2}$, then to minimize the total cost, $y_{a}$ and $y_{b}$ should be chosen such that $q_{a}$ and $q_{b}$ satisfy

$$
\begin{aligned}
\frac{1}{1-q_{b}}-\frac{w_{b}}{\beta_{b} q_{a}^{2}} & =0, \\
\frac{q_{a}}{\left(1-q_{b}\right)^{2}}-\frac{w_{a}}{\beta_{a} q_{b}^{2}} & =0,
\end{aligned}
$$

and the initial state is $p_{a}^{*}=\frac{q_{a}}{1-q_{b}}, p_{b}^{*}=0$.

Proof: Notice that minimization problem (11) can be considered as a subproblem of minimization problem (14). Given $\lambda_{1}=\lambda_{2}=1$ and $\gamma_{1}=\gamma_{2}=1$, from Lemma 1,

$$
C_{\min }= \begin{cases}\frac{q_{1}}{1-q_{2}} & \text { if } q_{1} \leq q_{2}, \\ \frac{q_{2}}{1-q_{1}} & \text { if } q_{1} \geq q_{2} .\end{cases}
$$

The minimum total cost can be obtained by solving

$$
\begin{array}{ll}
\text { minimize } & \frac{q_{a}}{1-q_{b}}+\frac{w_{a}}{\beta_{a} q_{b}}+\frac{w_{b}}{\beta_{b} q_{a}} \\
\text { subject to } & 0 \leq q_{a} \leq q_{b} \leq \frac{1}{2} .
\end{array}
$$

The minimum can be obtained by setting the derivatives of the objective function with respect to $q_{a}$ and $q_{b}$ to zero. Therefore we get (15) and (16), and hence $\frac{w_{b} / \beta_{b}}{\left(w_{a} / \beta_{a}\right)^{2}}=$ $\frac{\left(1-q_{b}\right)^{3}}{q_{b}^{4}}$. For $0 \leq q_{b} \leq \frac{1}{2}, \frac{\left(1-q_{b}\right)^{3}}{q_{b}^{4}}$ is strictly decreasing in $q_{b}$ and its minimum is 2 , therefore $\frac{w_{b} / \beta_{b}}{\left(w_{a} / \beta_{a}\right)^{2}} \geq 2$ guarantees a unique $q_{b}$ such that $0 \leq q_{b} \leq \frac{1}{2}$. If $\frac{w_{b}}{\beta_{b}} \leq \frac{1}{2}$ and $0 \leq q_{b} \leq \frac{1}{2}$, then from (15), $\frac{q_{a}}{q_{b}}=\sqrt{\frac{w_{b}}{\beta_{b}} \frac{1-q_{b}}{q_{b}^{2}}} \leq 1$. Hence, $q_{a}$ and $q_{b}$ obtained from solving (15) and (16) satisfy all constraints in minimization problem (17) and solve (17).

\section{PRofit MAXIMIZATION}

Assume the innovator obtains a revenue of $r_{a}$ per unit time from each type- $a$ player who adopts it. Assume the innovator discounts future revenue at a rate of $\theta$ to account for depreciation. Hence, the total discounted revenue is given by

$$
R\left(p_{1}, p_{2}\right)=\int_{0}^{\infty}\left(r_{1} p_{1}(t)+r_{2} p_{2}(t)\right) e^{-\theta t} d t,
$$

where $\left(p_{1}(t), p_{2}(t)\right)$ is the state at time $t$ if the initial state is $\left(p_{1}, p_{2}\right)$. Assume a linear advertisement cost $C\left(p_{1}, p_{2}\right)=$ $\gamma_{1} p_{1}+\gamma_{2} p_{2}$ as in Section V-A. The net profit of the innovator is the total discounted revenue less advertisement cost:

$$
\begin{aligned}
P\left(p_{1}, p_{2}\right) & =R\left(p_{1}, p_{2}\right)-C\left(p_{1}, p_{2}\right) \\
& =\int_{0}^{\infty}\left(r_{1} p_{1}(t)+r_{2} p_{2}(t)\right) e^{-\theta t} d t-\gamma_{1} p_{1}-\gamma_{2} p_{2} .
\end{aligned}
$$

How should the innovator choose $p_{1}$ and $p_{2}$ so that its net profit is maximized? Here we do not require the initial state to be inside the basin of attraction of $(1,1)$, so the innovator only takes over the market if it is profitable to do so.

By substituting $p_{1}(t)$ and $p_{2}(t)$ given in Theorem 1 into (18), we obtain the total discounted revenue as follows.

Lemma 3: If the initial state is $\left(p_{1}, p_{2}\right)$, the total discounted revenue is given by

1) in the case of $p_{1} \geq q_{1}$ and $p_{2} \geq q_{2}$ :

$$
\begin{aligned}
R\left(p_{1}, p_{2}\right)= & \frac{r_{1}}{\lambda_{1}+\theta} p_{1}+\frac{r_{2}}{\lambda_{2}+\theta} p_{2} \\
& +\frac{r_{1} \lambda_{1}}{\theta\left(\lambda_{1}+\theta\right)}+\frac{r_{2} \lambda_{2}}{\theta\left(\lambda_{2}+\theta\right)} ;
\end{aligned}
$$

2) in the case of $p_{a} \geq q_{a}$ and $p_{b}<q_{b}$ :

a) if $\frac{1}{\lambda_{a}} \ln \frac{p_{a}}{q_{a}} \geq \frac{1}{\lambda_{b}} \ln \frac{1-p_{b}}{1-q_{b}}$ :

$$
\begin{aligned}
R\left(p_{1}, p_{2}\right)= & \frac{r_{a}}{\lambda_{a}+\theta} p_{a}+\frac{r_{b}}{\lambda_{b}+\theta} p_{b}+\frac{r_{b} \lambda_{b}}{\theta\left(\lambda_{b}+\theta\right)} \\
& +\frac{r_{a} \lambda_{a}}{\theta\left(\lambda_{a}+\theta\right)}\left(\frac{1-q_{b}}{1-p_{b}}\right)^{\frac{\theta}{\lambda_{b}}} ;
\end{aligned}
$$

b) if $\frac{1}{\lambda_{a}} \ln \frac{p_{a}}{q_{a}}<\frac{1}{\lambda_{b}} \ln \frac{1-p_{b}}{1-q_{b}}$ :

$$
\begin{aligned}
R\left(p_{1}, p_{2}\right)= & \frac{r_{a}}{\lambda_{a}+\theta} p_{a}+\frac{r_{b}}{\lambda_{b}+\theta} p_{b}+\frac{r_{b} \lambda_{b}}{\theta\left(\lambda_{b}+\theta\right)} \\
& -\frac{r_{b} \lambda_{b}}{\theta\left(\lambda_{b}+\theta\right)}\left(\frac{q_{a}}{p_{a}}\right)^{\frac{\theta}{\lambda_{a}}} ;
\end{aligned}
$$

$3)$ in the case of $p_{1}<q_{1}$ and $p_{2}<q_{2}$ :

$$
R\left(p_{1}, p_{2}\right)=\frac{r_{1}}{\lambda_{1}+\theta} p_{1}+\frac{r_{2}}{\lambda_{2}+\theta} p_{2} .
$$

The total discounted revenue is not continuous because of the phase transition described in Theorem 1. Also, the total discounted revenue can be regarded as a sum of three terms, the first only depends on $p_{1}$, the second only depends on $p_{2}$, and the remaining one is a constant. Therefore, the net profit also has these properties, and can be maximized 
by considering $p_{1}$ and $p_{2}$ separately. The following result is obtained based on these observations.

Theorem 5: The initial state maximizing the net profit is given as follows:

1) in the case of $\frac{r_{1}}{\lambda_{1}+\theta}>\gamma_{1}$ and $\frac{r_{2}}{\lambda_{2}+\theta}>\gamma_{2}:(1,1)$;

2) in the case of $\frac{r_{a}^{+}}{\lambda_{a}+\theta}=\gamma_{a}$ and $\frac{\lambda_{b}}{\lambda_{b}+\theta}>\gamma_{b}: p_{b}^{*}=1$ and any $p_{a}^{*}$ such that $q_{a} \leq p_{a}^{*} \leq 1$;

$3)$ in the case of $\frac{r_{1}}{\lambda_{1}+\theta}=\gamma_{1}$ and $\frac{r_{2}}{\lambda_{2}+\theta}=\gamma_{2}:\left(p_{1}^{*}, p_{2}^{*}\right)$ for $q_{1} \leq p_{1}^{*} \leq 1$ and $q_{2} \leq p_{2}^{*} \leq 1$

4) in the case of $\frac{r_{a}}{\lambda_{a}+\theta}<\gamma_{a}$ and $\frac{r_{b}}{\lambda_{b}+\theta}>\gamma_{b}$ :

a) if $\frac{\lambda_{a}}{\lambda_{b}}<\frac{\ln \left(1-q_{a}\right)}{\ln q_{b}}: p_{b}^{*}=1$, and the maximizing $p_{a}^{*}$ jumps from $q_{a}$ to $1-\left(1-q_{a}\right) q_{b}^{-\frac{\lambda_{a}}{\lambda_{b}}}$, and to 0 , as $\gamma_{a}$ increases;

b) if $\frac{\lambda_{a}}{\lambda_{b}} \geq \frac{\ln \left(1-q_{a}\right)}{\ln q_{b}}: p_{b}^{*}=1$, and the maximizing $p_{a}^{*}$ jumps from $q_{a}$ to 0 , as $\gamma_{a}$ increases;

5) in the case of $\frac{r_{a}}{\lambda_{a}+\theta}<\gamma_{a}$ and $\frac{r_{b}}{\lambda_{b}+\theta}=\gamma_{b}$ :

a) if $\frac{\lambda_{a}}{\lambda_{b}}<\frac{\ln \left(1-q_{a}\right)}{\ln q_{b}}$ : the maximizing state jumps from $p_{a}^{*}=q_{a}$ and any $p_{b}^{*}$ such that $q_{b} \leq p_{b}^{*} \leq 1$ to $p_{a}^{*}=$ $1-\left(1-q_{a}\right) q_{b}^{-\frac{\lambda_{a}}{\lambda_{b}}}$ and $p_{b}^{*}=1$, and to $p_{a}^{*}=0$ and any $p_{b}^{*}$ such that $q_{b} \leq p_{b}^{*} \leq 1$, as $\gamma_{a}$ increases;

b) if $\frac{\lambda_{a}}{\lambda_{b}} \geq \frac{\ln \left(1-q_{a}\right)}{\ln q_{b}}$ : the maximizing state jumps from $p_{a}^{*}=q_{a}$ and any $p_{b}^{*}$ such that $q_{b} \leq p_{b}^{*} \leq 1$ to $p_{a}^{*}=0$ and any $p_{b}^{*}$ such that $q_{b}\left(1-q_{a}\right)^{-\frac{\lambda_{b}}{\lambda_{a}}} \leq p_{b}^{*} \leq 1$, as $\gamma_{a}$ increases;

6) in the case of $\frac{r_{1}}{\lambda_{1}+\theta}<\gamma_{1}$ and $\frac{r_{2}}{\lambda_{2}+\theta}<\gamma_{2}$ : the state maximizing the net profit can be $(0,0)$ for large $\gamma_{1}$ and $\gamma_{2}$, any point lying on (4) for small $\gamma_{1}$ and $\gamma_{2}$, or

a) if $\frac{\lambda_{1}}{\lambda_{2}}<\frac{\ln \left(1-q_{1}\right)}{\ln q_{2}}$ : $\left(p_{1}^{*}, 0\right)$ where $q_{1}<p_{1}^{*}<q_{1}(1-$ $\left.q_{2}\right)^{-\frac{\lambda_{1}}{\lambda_{2}}}$ for small $\gamma_{1}$ and large $\gamma_{2}$, or $\left(0, p_{2}^{*}\right)$ where $q_{2}<p_{2}^{*} \leq 1$ for large $\gamma_{1}$ and small $\gamma_{2}$;

b) if $\frac{\ln \left(1-q_{1}\right)}{\ln q_{2}} \leq \frac{\lambda_{1}}{\lambda_{2}} \leq \frac{\ln q_{1}}{\ln \left(1-q_{2}\right)}:\left(p_{1}^{*}, 0\right)$ where $q_{1}<$ $p_{1}^{*}<q_{1}\left(1-q_{2}\right)^{-\frac{\lambda_{1}}{\lambda_{2}}}$ for small $\gamma_{1}$ and large $\gamma_{2}$, or $\left(0, p_{2}^{*}\right)$ where $q_{2}<p_{2}^{*}<q_{2}\left(1-q_{1}\right)^{-\frac{\lambda_{2}}{\lambda_{1}}}$ for large $\gamma_{1}$ and small $\gamma_{2}$;

c) if $\frac{\lambda_{1}}{\lambda_{2}}>\frac{\ln q_{1}}{\ln \left(1-q_{2}\right)}:\left(p_{1}^{*}, 0\right)$ where $q_{1}<p_{1}^{*} \leq 1$ for small $\gamma_{1}$ and large $\gamma_{2}$, or $\left(0, p_{2}^{*}\right)$ where $q_{2}<p_{2}^{*}<$ $q_{2}\left(1-q_{1}\right)^{-\frac{\lambda_{2}}{\lambda_{1}}}$ for large $\gamma_{1}$ and small $\gamma_{2}$.

Proof: From Lemma 3, the revenue is increasing in both $p_{1}$ and $p_{2}$. If $\frac{r_{1}}{\lambda_{1}+\theta} \geq \gamma_{1}$ and $\frac{r_{2}}{\lambda_{2}+\theta} \geq \gamma_{2}$, then the net profit is also increasing in both $p_{1}$ and $p_{2}$, and the maximum net profit is attained at $\left(p_{1}, p_{2}\right)=(1,1)$. Since in the region $p_{1} \geq q_{1}$ and $p_{2} \geq q_{2}$, the net profit is independent of $p_{a}$ if $\frac{r_{a}}{\lambda_{a}+\theta}=\gamma_{a}$, we get the first three results in the theorem.

If $\frac{r_{b}}{\lambda_{b}+\theta}>\gamma_{b}$, the net profit is increasing in $p_{b}$ and the maximum can only be attained if $p_{b}=1$. Given $p_{b}^{*}=1$, if $\frac{r_{a}}{\lambda_{a}+\theta}<\gamma_{a}$, there are two cases to consider.

1) If $\frac{\lambda_{a}}{\lambda_{b}} \geq \frac{\ln \left(1-q_{a}\right)}{\ln q_{b}}$, the net profit is decreasing in $p_{a}$ if $p_{a} \geq q_{a}$, and is convex in $p_{a}$ if $p_{a} \leq q_{a}$. Therefore, the maximum net profit can be attained only at either $p_{a}=q_{a}$ or $p_{a}=0$.

2) If $\frac{\lambda_{a}}{\lambda_{b}}<\frac{\ln \left(1-q_{a}\right)}{\ln q_{b}}$, the net profit is decreasing in $p_{a}$ if $p_{a} \geq q_{a}$ or $p_{a}<1-\left(1-q_{a}\right) q_{b}^{-\frac{\lambda_{a}}{\lambda_{b}}}$, and is convex in $p_{a}$ if $1-\left(1-q_{a}\right) q_{b}^{-\frac{\lambda a}{\lambda_{b}}} \leq p_{a} \leq q_{a}$. Since the net profit is discontinuous at $p_{a}=1-\left(1-q_{a}\right) q_{b}^{-\frac{\lambda_{a}}{\lambda_{b}}}, p_{b}=1$, the maximum net profit can be attained at either $p_{a}=q_{a}$, $p_{a}=1-\left(1-q_{a}\right) q_{b}^{-\frac{\lambda_{a}}{\lambda_{b}}}$ or $p_{a}=0$.

The case of $\frac{r_{b}}{\lambda_{b}+\theta}=\gamma_{b}$ and $\frac{r_{a}}{\lambda_{a}+\theta}<\gamma_{a}$ is almost the same as the case just described, except that when $p_{a}=q_{a}$, the net profit is the same for any $p_{b}$ such that $q_{b} \leq p_{b} \leq 1$; and when $p_{a}=0$, the net profit is the same for any $p_{b}$ such that $q_{b}\left(1-q_{a}\right)^{-\frac{\lambda_{b}}{\lambda_{a}}} \leq p_{b} \leq 1$ if $\frac{\lambda_{a}}{\lambda_{b}} \geq \frac{\ln \left(1-q_{a}\right)}{\ln q_{b}}$, or for any $p_{b}$ such that $q_{b} \leq p_{b} \leq 1$ if $\frac{\lambda_{a}}{\lambda_{b}}<\frac{\ln \left(1-q_{a}\right)}{\ln q_{b}}$.

For the last case (6):

- If $p_{1} \geq q_{1}$ and $p_{2} \geq q_{2}$, the net profit is decreasing in both $p_{1}$ and $p_{2}$, therefore the maximum net profit can only be attained at $\left(q_{1}, q_{2}\right)$, which lies on (4).

- If $p_{1}<q_{1}$ and $p_{2}<q_{2}$, the net profit is also decreasing in both $p_{1}$ and $p_{2}$, therefore the maximum net profit can only be attained at $(0,0)$.

- If $p_{a} \geq q_{a}, p_{b} \leq q_{b}$ and $\frac{1}{\lambda_{a}} \ln \frac{p_{a}}{q_{a}} \geq \frac{1}{\lambda_{b}} \ln \frac{1-p_{b}}{1-q_{b}}$, the net profit is decreasing in $p_{a}$ and convex in $p_{b}$, the maximum net profit can only be attained on (4).

- If $p_{a} \geq q_{a}, p_{b} \leq q_{b}$ and $\frac{1}{\lambda_{a}} \ln \frac{p_{a}}{q_{a}}<\frac{1}{\lambda_{b}} \ln \frac{1-p_{b}}{1-q_{b}}$, the net profit is concave in $p_{a}$ and decreasing in $p_{b}$, the maximum net profit can only be attained at $p_{b}=0$ and $p_{a}$ such that $q_{a}<p_{a}<q_{a}\left(1-q_{b}\right)^{-\frac{\lambda_{a}}{\lambda_{b}}}$ if $\frac{\lambda_{a}}{\lambda_{b}} \leq$ $\frac{\ln q_{a}}{\ln \left(1-q_{b}\right)}$, or $p_{b}=0$ and $p_{a}$ such that $q_{a}<p_{a} \leq 1$ if $\frac{\lambda_{a}}{\lambda_{b}}>\frac{\ln q_{a}}{\ln \left(1-q_{b}\right)}$.

Theorem 5 states four possibilities when the maximum net profit is attained.

1) If the maximizing state is $(0,0)$ (when $\frac{r_{1}}{\lambda_{1}+\theta}<\gamma_{1}$ and $\left.\frac{r_{2}}{\lambda_{2}+\theta}<\gamma_{2}\right)$, the innovator should not enter the market.

2 ) If the maximizing state is $\left(p_{1}, 0\right)$ or $\left(0, p_{2}\right)$ in the basin of attraction of $(0,0)$ (when $\frac{r_{1}}{\lambda_{1}+\theta}<\gamma_{1}$ and $\frac{r_{2}}{\lambda_{2}+\theta}<$ $\gamma_{2}$ ), the innovator should enter the market, but should not take over the market.

3) If the maximizing state is $(1,1)$ (when $\frac{r_{1}}{\lambda_{1}+\theta} \geq \gamma_{1}$ and $\frac{r_{2}}{\lambda_{2}+\theta} \geq \gamma_{2}$ ), the innovator needs advertisements only to take over the market.

4) In all other cases, the innovator needs both advertisements and interaction between players to take over the market.

Fig. 3 illustrates all possible states that maximize the net profit.

\section{CONCLUSION}

We have considered diffusion of innovation in two-sided markets. Conditions on the initial state for the innovation to spread to the entire market have been obtained. We also solved three related optimization problems when the innovator can devote some resources to advertising and technology improvement. The solutions provide guidelines on selecting the initial state.

\section{REFERENCES}

[1] J.-C. Rochet and J. Tirole, "Platform competition in two-sided markets," Journal of the European Economic Association, vol. 1, no. 4, pp. 990-1029, Jun. 2003. 


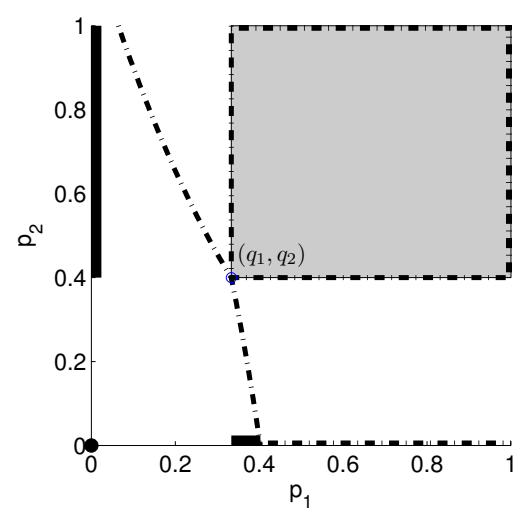

(a)

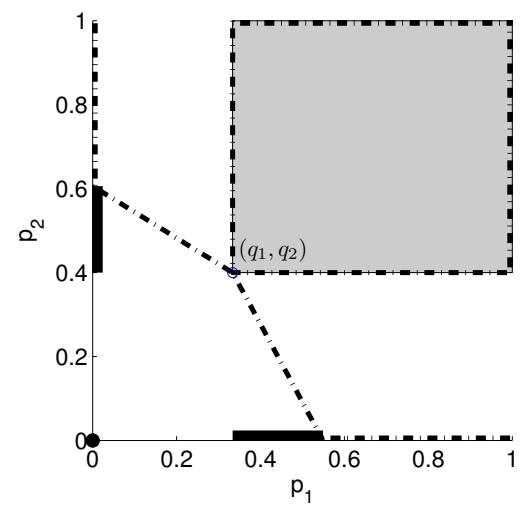

(b)

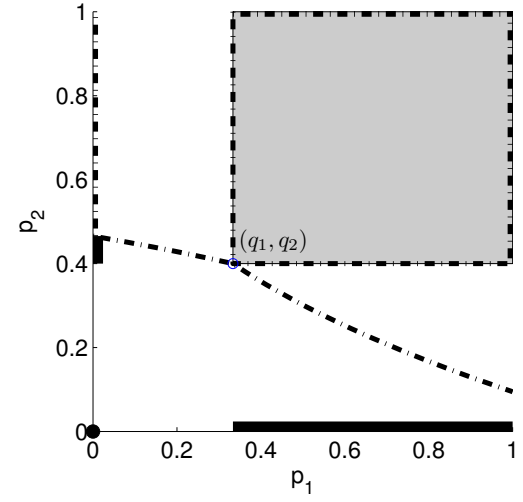

(c)

Fig. 3. Net profit maximization when $q_{1}=\frac{1}{3}, q_{2}=\frac{2}{5}$ and (a) $\frac{\lambda_{1}}{\lambda_{2}}<\frac{\ln \left(1-q_{1}\right)}{\ln q_{2}}$, (b) $\frac{\ln \left(1-q_{1}\right)}{\ln q_{2}} \leq \frac{\lambda_{1}}{\lambda_{2}} \leq \frac{\ln q_{1}}{\ln \left(1-q_{2}\right)}$, and (c) $\frac{\lambda_{1}}{\lambda_{2}}>\frac{\ln q_{1}}{\ln \left(1-q_{2}\right)}$. Evolution from any initial state on dash-dotted line or in the shaded area reaches $(1,1)$, while evolution from any initial state on solid line or dot reaches $(0,0)$.

[2] T. Eisenmann, G. G. Parker, and M. W. van Alstyne, "Strategies for two-sided markets," Harvard Business Review, Oct. 2006.

[3] M. Kandori, G. J. Mailath, and R. Rob, "Learning, mutation, and long run equilibria in games," Econometrica, vol. 61, no. 1, pp. 29-56, Jan. 1993.

[4] L. E. Blume, "The statistical mechanics of strategic interaction," Games and Economic Behavior, vol. 5, no. 3, pp. 387-424, Jul. 1993.

[5] G. Ellison, "Learning, local interaction, and coordination," Econometrica, vol. 61, no. 5, pp. 1047-1071, Sep. 1993.

[6] A. Montanari and A. Saberi, "The spread of innovations in social networks," PNAS, vol. 108, no. 41, pp. 20 196-20 201, Nov. 2010.

[7] G. E. Kreindler and H. P. Young, "Fast convergence in evolutionary equilibrium selection," Oxford, Tech. Rep. Oxford Economics Discussion Paper 569, Oct. 2011.

[8] S. Morris, "Contagion," Review of Economic Studies, vol. 67, no. 1, pp. 57-78, Jan. 2000.

[9] L. E. Blume, "The statistical mechanics of best-response strategy revision," Games and Economic Behavior, vol. 11, no. 2, pp. 111145, Nov. 1995.

[10] D. López-Pintado, "Contagion and coordination in random networks," International Journal of Game Theory, vol. 34, no. 3, pp. 371-381, Oct. 2006.

[11] M. O. Jackson and L. Yariv, "Diffusion of behavior and equilibrium properties in network games," American Economic Review, vol. 97, no. 2, pp. 92-98, May 2007.

[12] H. P. Young, "Innovation diffusion in heterogeneous populations: Contagion, social influence, and social learning," American Economic Review, vol. 99, no. 5, pp. 1899-1924, Dec. 2009.

[13] N. Immorlica, J. Kleinberg, M. Mahdian, and T. Wexler, "The role of compatibility in the diffusion of technologies through social networks," in Proceedings of the 8th ACM conference on Electronic commerce (EC '07), San Diego, CA, USA, Jun. 2007.

[14] D. Acemoglu, A. Ozdaglar, and E. Yildiz, "Diffusion of innovations in social networks," in IEEE Conference on Decision and Control (CDC), Orlando, FL, USA, Dec. 2011.

[15] M. Lelarge. (2011, Oct.) Diffusion and cascading behavior in random networks. [Online]. Available: http://arxiv.org/abs/1012.2062

[16] M. Kearns, "Graphical games," in Algorithmic Game Theory, N. Nisan, T. Roughgarden, E. Tardos, and V. V. Vazirani, Eds. Cambridge University Press, Dec. 2007, ch. 7.

[17] E. M. Rogers, Diffusion of Innovations, 4th ed. The Free Press, 1995.

[18] R. Kumar, Y. Lifshits, and A. Tomkins, "Evolution of two-sided markets," in Third ACM International Conference on Web Search and Data Mining (WSDM '10), New York, NY, USA, Feb. 2010.

[19] M. Staudigl, "Stochastic stability in asymmetric binary choice coordination games," Games and Economic Behavior, vol. 75, no. 1, pp. 372-401, May 2012.

[20] S. Aral, L. Muchnik, and A. Sundararajan. (2011, Feb.) Engineering social contagions: Optimal network seeding and incentive strategies. [Online]. Available: http://ssrn.com/abstract=1770982
[21] M. Armstrong, "Competition in two-sided markets," RAND Journal of Economics, vol. 37, no. 3, pp. 667-691, Sep. 2006.

[22] J. C. Harsanyi and R. Selton, A General Theory of Equilibrium Selection in Games. MIT Press, 1988.

[23] S. N. Ethier and T. G. Kurtz, Markov Processes: Characterization and Convergence, 2nd ed. John Wiley \& Sons, 2005. 